\title{
Some Point-Wise Estimates for Solutions of a Class of Nonlinear Functional-Integral Inequalities.
}

\author{
JAGDish Chandra (Durham, North Carolina, U.S.A.) (*) \\ V. LaKshMtKaNtham (Kingston, Rhode Island, U.S.A.)
}

\begin{abstract}
Summary. - The celebrated Gronwall-Bellman Lemma provides explicit bounds on solutions of a elass of linear integral inequalities. The aim of this paper is to prove sufficiently general results analogous to this Lemma for functional-integral inequalities. These results are useful for obtaining point-wise estimates or comparison theorems for solutions of functional differential equations and functional-integral equations of Volterra type.
\end{abstract}

\section{1. - Introduction.}

There exist various generalizations of Gronwall-Reid-Bellman inequalities. These generalizations are motivated by specific applications to the theory of ordinary differential equations. For instance, in obtaining point-wise estimates or bounds on the norm of solutions, in establishing uniqueness of solutions or in deriving conditions for stability of solutions, such inequalities are handy tools $[9,10]$. The aim of this paper is to prove sufficiently general results in this direction involving functional-integral inequalities. The results on functional-integral inequalities (Section 2) are useful for obtaining point-wise estimates or comparison theorems for solutions of functional differential equations and functional-integral equations of Volterra type. Evidently, our results in Section 3 include and unify some recent generalizations $[2,5,6]$ of the Gronwall type inequalities.

The celebrated Gronwall-Bellman lemma provides explicit bounds on solutions of a class of linear integral inequalities. In recent years there have been several linear and nonlinear generalizations of this useful lemma (for instance, see the survey paper [3]). In Section 2 of this paper we present an analogous result for a class of nonlinear functional-integral inequalities. This result, which may be of interest by itself, serves as a basis for a further generalization in Section 3. Some useful lemmas are derived in Section 4. These together with the main result in Section 3 lead to the recovery and improvement of some recent generalizations of the Gronwall type inequalities. This is accomplished in the final Section 5 .

\section{2. - Functional-integral inequalities.}

Consider the functional-integral inequality

$$
x(t) \leqslant c(t)+\int_{t_{0}}^{t} k(t, s) \omega(s, x(\varphi(s))) d s .
$$

$\left({ }^{*}\right)$ Entrata in Redazione il $1^{\circ}$ settembre 1971. 
Existence and properties of solutions of the functional-integral equation

$$
x(t)=c\left(t_{0}\right)+\int_{t_{0}}^{t} k(t, s) \omega(s, x(\varphi(s))) d s
$$

have been investigated, among others, by Cordunkanu [4]. The equation (2.2) can be considered as a generalization of the functional-differential equations of the type

$$
\dot{x}(t)=A(t) x(t)+\omega(t, x(\varphi(t)))
$$

with suitable initial conditions. Such equations arise in a natural way in the study of feedback control systems. Equation (2.2) is also related to the study of boundary value problems associated with functional-differential equations [7]. Here we shall restrict ourselves to obtaining point-wise bounds on $x(t)$.

Let $I$ denote the closed interval $t_{0} \leqslant t \leqslant T$ and let $E_{t_{0}}$ denote the set

$$
E_{i_{0}}=t_{0} D\left\{\varphi(t) \mid \varphi(t) \leqslant t_{0} \text { for } t \in I\right\} \text {. }
$$

Suppose $\mathcal{H}$ denotes the Banach space of continuous functions defined on $E_{t_{0}} D I$ with the norm

$$
x(t) \in \mathcal{M}, \quad\|x\|=\operatorname{Sup}_{\substack{\mathbb{E}_{t_{0}} D t \\ \text { D. }}}|x(t)|
$$

Let $\pi \subset \mathcal{M}$ denote the closed, convex cone of non-negative functions in $\mathcal{M}$. For any $c(t) \in \mathcal{K}$, let $S$ denote those elements in $\mathcal{K}$ which coincide with $c(t)$ on $E_{t_{i}}$. We shall always assume that $\varphi(t)$ is a continuous function defined on a suitable interval and satisfy $\varphi(t) \leqslant t$. We shall make the following hypotheses:

$\left(\mathrm{H}_{1}\right) k(t, s)$ is non-negative and continuous on $0 \leqslant s \leqslant t \leqslant T$; for each $s, k(t, s)$ is non-decreasing in $t$.

$\left(\mathrm{H}_{2}\right) \omega: I \times R^{+} \rightarrow I \times R^{+}$is continuous.

$\left(\mathbf{H}_{3}\right) \omega(t, x)$ is non-decreasing in $x$ for each $t \in I$.

$\left(\mathrm{H}_{4}\right)$ There exists a $\varrho>0$ such that $\|c(t)\| \leqslant \varrho$ and

$$
c\left(t_{0}\right)+\int_{t_{0}}^{T} k(t, s) \omega(s, o) d s \leqslant \varrho .
$$

Define

$$
B_{\varrho}=\{x \in S \mid\|x\| \leqslant \varrho\}
$$

The following theorem gives a constructive existence proof of the maximal solution of $(2.2)$ in $S_{e}$. 
THEOREM 1. - Let $\left(\mathbf{H}_{1}\right)-\left(\mathbf{H}_{4}\right)$ hold. Define the iterates

$$
y_{n}(t)=o\left(t_{0}\right)+\int_{t_{0}}^{t} k(t, s) \omega\left(s, y_{n-1}(\varphi(s))\right) d s, \quad t \in I
$$

where

$$
y_{0}(t)=c\left(t_{0}\right)+\int_{i_{0}}^{t} k(t, s) \omega(s, \varrho) d s .
$$

Then the sequence $\left\{y_{n}(t)\right\}$ converges uniformly to the maximal solution of $(2.2)$ in $S_{\varrho}$.

Proof. - By $\left(\mathrm{H}_{4}\right), y_{0}(t) \in S_{\varrho}$. Now using $\left(\mathrm{H}_{3}\right)$

$$
y_{1}(t)=c\left(t_{0}\right)+\int_{i_{0}}^{t} k(t, s) \omega\left(s, y_{0}(\varphi(s))\right) d s \leqslant c\left(t_{0}\right)+\int_{t_{0}}^{t} k(t, s) \omega(s, \varrho) d s=y_{0}(t) .
$$

Here we have used the fact that

$$
y_{0}(\varphi(t))=c\left(t_{0}\right)+\int_{t_{0}}^{\varphi(t)} k(\varphi(t), s) \omega(s, \varrho) d s \leqslant c\left(t_{0}\right)+\int_{t_{0}}^{t} k(t, s) \omega(s, \varrho) d s \leqslant \varrho .
$$

Using an inductive argument it is easily seen that

$$
\varrho \geqslant y_{0}(t) \geqslant y_{1}(t) \geqslant \ldots \geqslant y_{n}(t) \geqslant y_{1+n}(t)
$$

Next, for any $n$ and $t^{\prime}, t^{\prime \prime} \in I$,

$$
\left\|y_{n}\left(t^{\prime}\right)-y_{n}\left(t^{\prime \prime}\right)\right\| \leqslant \int_{t_{0}}^{t^{\prime}}\left\|k\left(t^{\prime}, s\right)-k\left(t^{\prime \prime}, s\right)\right\|\left\|\omega\left(s, y_{n-1}\right)\right\| d s+\int_{t^{\prime}}^{t^{\prime \prime}}\left\|k\left(t^{\prime \prime}, s\right)\right\|\left\|\omega\left(s, y_{n-1}\right)\right\| d s .
$$

Therefore from the uniform continuity of $k(t, s)$ on any closed interval, it is readily seen that $\left\{y_{n}(t)\right\}$ is equi-continuous. Hence by Arzela's Theorem $\left\{y_{n}(t)\right\}$ is precompact and being monotonic converges uniformly to $y(t) \in S_{\mathrm{e}}$ [8]. From the continuity of $\omega, y(t)$ is a solution of $(2.2)$.

Now, let $x(t)$ be any other solution of $(2.2)$ in $\delta_{g}$. Then

$$
y_{0}(t)-x(t)=\int_{t_{0}}^{t} \bar{k}(t, s)[f(s, \varrho)-f(x(\varphi(s)))] d s \geqslant 0 .
$$

In fact, induction shows that for each $n$

$$
y_{n}(t)-\infty(t) \geqslant 0
$$

Therefore, in the limit, $y(t)-x(t) \geqslant 0$. This completes the proof of Theorem 1.

$$
5 \text { - Annali di Matematica }
$$


From Theorem 1, we derive the following comparison result.

CoRollary 1. - Let $x(t) \in S_{e}$ satisfy

$$
x(t) \leqslant c\left(t_{0}\right)+\int_{i_{0}}^{t} k(t, s) \omega(s, x(\varphi(s))) d s, \quad t \in I .
$$

Then under the assumptions of Theorem 1.

$$
x(t) \leqslant y(t)
$$

where $y(t)$ is the maximal solution of $(2,2)$ in $\mathbb{S}_{e}$ for $t \in I$.

Proof. - Define the iterates

$$
Z_{n}(t)=c\left(t_{0}\right)+\int_{t_{0}}^{t} k(t, s) \omega\left(s, Z_{n-1}(\varphi(s))\right) d s, \quad t \in I,
$$

where

$$
Z_{0}(t)=x(t) \in S_{e}
$$

Then

$$
Z_{1}(t)=o\left(t_{0}\right)+\int_{t_{0}}^{t} k(t, s) \omega(s, x(\varphi(s))) \geqslant x(t), \quad t \in T
$$

By an inductive argument, using monotonicity of $\omega$,

$$
x(t) \leqslant Z_{1}(t) \leqslant \ldots \leqslant Z_{n}(t) \leqslant \cdots
$$

But $\left\{\left(Z_{n}(t)\right\}\right.$ converges to a solution $Z(t)$ of $(2.2)$ in $S_{e}$.

The maximality of $y(t)$ then implies $Z(t) \leqslant y(t)$. This completes the proof.

REMARK 1. - Corollary 1 generalizes Theorem 1.9 .2 in [9].

Now let us set $u(t)=x(t)-c(t)$, then $u(t)$ satisfies the functional-integral inequality

$$
u(t) \leqslant \int_{t_{0}}^{t} k(t, s) \omega(s, c(\varphi(s))+u(\varphi(s))) d s=\int_{t_{0}}^{t} k(t, s) \tilde{\omega}(s, u(\varphi(s))) d s
$$

It is easy to verify that $\tilde{\omega}(t, \infty)$ satisfies $\left(\mathbf{H}_{2}\right)$ and $\left(\mathbf{H}_{3}\right)$.

$\left(\mathrm{H}_{4}^{\prime}\right)$ There exists a $\varrho>0$ such that

$$
\int_{t_{0}}^{F} k(t, s) \tilde{\omega}(s, \varrho) d s \leqslant \varrho
$$


Under the hypothesis of Theorem 1 where $\left(\mathbf{H}_{4}\right)$ is replaced by $\left(\mathbf{H}_{4}^{\prime}\right)$, the maximal solution for the functional-integral equation

$$
u(t)=\int_{t_{0}}^{t} k(t, s) \tilde{\omega}(s, u(\varphi(s))) d s
$$

exists in $S_{\varrho}$. Thus we are led to

THEOREM 2. - Let $\left(\mathrm{H}_{1}\right)-\left(\mathrm{H}_{3}\right)$ and $\left(\mathrm{H}_{4}^{\prime}\right)$ hold. If $x(t) \in S_{Q}$ satisfies $(2.1)$ then $x(t) \leqslant$ $\leqslant c(t)+y(t)$, where $y(t)$ is the maximal solution of $(2.4)$ in $s_{\varrho}$.

Remari 2. - Theorem 2 generalizes Corollary 1.9.4 in [9].

\section{3. - A further generalization.}

Let us now consider the functional-integral inequality

$$
f(x(t)) \leqslant a(t)+b(t) h\left[o(t)+\int_{t_{0}}^{t} 7 c(t, s) \omega(s, x(p(s))) d s\right]
$$

where $a, b, c \in \pi$, and $f$ and $h$ map continuously $R^{+}$onto $R^{+}$. Further, let $f$ be strictly increasing and $h$ be non-decreasing.

By setting

$$
r(t)=c(t)+\int_{t_{0}}^{t} k(t, s) \omega(s, x(\varphi(s))) d s
$$

in (3.1), we immediately get

$$
x(t) \leqslant f^{-1}[a(t)+b(t) h(r(t))] .
$$

Substitution of (3.3) in (3.2) yields

$$
\begin{aligned}
& r(t) \leqslant c(t)+\int_{t_{a}}^{t} k(t, s) \omega\left(s, f^{-1}[a(\varphi(s))+b(\varphi(s)) h(r(\varphi(s)))]\right) d s= \\
&=c(t)+\int_{t_{0}}^{t} k(t, s) W(s, r(\varphi(s))) d s .
\end{aligned}
$$

where we have set $W(t, u)=\omega\left(t, f^{-1}[a(t)+b(t) h(u(t))]\right)$.

From $\left(\mathrm{H}_{2}\right)$ and $\left(\mathrm{H}_{3}\right), W$ is continuous and non-decreasing in its second argument. This leads to the main result of this paper: 
Theorem 3. - Under the assumptions of Theorem 2 , if $x(t) \in S_{e}$ satisfies (3.1), then for $t \in I_{0}$

$$
x(t) \leqslant f^{-1}[a(t)+b(t) h(o(t)+R(t))],
$$

where $I_{0}=\left\{t \in I \mid[a(t)+b(t) h(c(t)+R(t))] \in \operatorname{Dom} f^{-1}\right\}$ and $R(t) \in S_{\mathrm{e}}$ is the maximal solution of the functional-integral equation

$$
r(t)=\int_{t_{0}}^{t} k(t, s) W(s, o(\varphi(s))+r(\varphi(s))) d s .
$$

REMARK 3. - Oontinuity assumptions on $k(t, s), a(t), b(t)$ and $e(t)$ can be relaxed considerably. However, in order to reduce some technical details, we shall refrain from doing this here.

REMarK 4. - Trivially, by setting $\varphi(t) \equiv t$, we shall bo able to generalize or recover several generalizations of Gronwall type inequalities. This will be accomplished in Section $\mathbf{5}$.

Before we embark on specializing Theorem 3, let us formulate another version of the theorem under some additional conditions. This form is found to be convenient in some applications.

Let us assume that $h(u)$ is strictly increasing $h^{-1}(v)$ is convex and submultiplicative for $v \in \nVdash$. Further, let $\alpha(t), \beta(t)$ belong to the interior of $\nVdash$ and satisfy $\alpha(t)+\beta(t)=1$. Then multiplying (3.1) by $h^{-1}$ and exploiting convexity and submultiplicity of $h^{-1}$, one gets

$$
F(x(t)) \leqslant m(t)+n(t)\left[c(t)+\int_{t_{0}}^{t} k(t, s) \omega(s, x(\varphi(s))) d s\right]
$$

where we have set

$$
F=h^{-1} f, m(t)=\alpha(t) h^{-1}\left(a(t)^{-1}\right), \quad n(t)=\beta(t) h^{-1}\left(b(t) \beta(t)^{-1}\right)
$$

An application of Theorem (3) to (3.7) then gives

THEOREM 4. - Let the assumptions of Theorem 3 hold. In addition, suppose $h$ is stricltly increasing, and $h^{-1}$ is convex and submultiplicative on the cone $\mathcal{K}$. Let $\alpha(t), \beta(t)$ belong to the interior of $\pi$ and satisfy $\alpha(t)+\beta(t)=1$. Now, if $x(t) \in S_{e}$ satisfies (3.1), then for $t \in I_{0}$,

$$
x(t) \leqslant F^{-1}[m(t)+n(t)\{c(t)+R(t)\}],
$$


where $I_{0}=\left\{t \in I \mid m(t)+n(t)[c(t)+R(t)] \in \operatorname{Dom} F^{-1}\right\}$ and $R(t)$ is the maximal solution of the functional-integral equation

$$
r(t)=\int_{t_{0}}^{t} k(t, s) \omega\left(s, F^{-1}[m(\varphi)+n(\varphi)\{c(\varphi)+r(\varphi)\}]\right) d s
$$

in $S_{Q}$ and $m(t), n(t)$ and $F^{-1}$ are given by (3.8).

\section{4. - Some useful lemmas.}

A very useful generalization of the Gronwall type inequalities is due to BrHARI [1] who derives explicit bounds on unknown function. Recently there have been several extensions of Bihari's result $[2,5,6]$. In Sections 2 and 3 we have presented sufficiently general results for nonlinear functional-integral inequalities where bounds were derived via maximal solutions of some related functional-integral equations. In the case of $\varphi(t) \equiv t$, we will now show that our bounds are sharper in the sense that in those cases where an explicit (Bihari Type) bound is available, either this bound coincides with our bound (obtained via maximal solution) or is larger than this. Thus our results not only generalize but sharpen some of these special results.

The following lemmas are derived under the additional assumptions that $\varphi(t) \equiv t$ and $\omega(t, u)=\omega(u)$.

Leina 1. - Let $R(t)$ be the maximal solution of

$$
r(t)=\int_{t_{0}}^{t} k(t, s) \omega F^{-1}[m(s)+n(s)\{c(s)+r(s)\}] d s
$$

existing on $I$. Then

$$
m(t)+n(t)[o(t)+R(t)] \leqslant \Omega^{-1}\left[\Omega(M(t)+N(t) C(t))+N(t) \int_{t_{0}}^{t} k(t, s) d s\right]
$$

on

where

$$
I_{0}=\left[t \in I: \Omega(M(t)+N(t) O(t))+N(t) \int_{i_{0}}^{t} k(t, s) d s \in \operatorname{Dom} \Omega^{-1}\right]
$$

$$
M(t)=\sup _{t_{0} \leqslant t \leqslant t} m(s), \quad N(t)=\sup _{t_{0} \leqslant s \leqslant t} n(s), \quad C(t)=\sup _{t_{0} \leqslant s \leqslant t} c(s)
$$

and

$$
\Omega(u)=\int_{\varepsilon}^{u} \frac{d s}{\omega F^{-1}(s)} d s, \quad \varepsilon, u>0
$$


Proof. - Let $R(t)$ be the maximal solution of (4.1). Set

$$
\nu(t)=m(t)+n(t)[c(t)+R(t)] .
$$

Then, clearly for $t_{0} \leqslant t \leqslant \sigma \leqslant T$, we have

$$
v(t) \leqslant \varrho(t)
$$

where

$$
\varrho(t)=M(\sigma)+N(\sigma)\left[O(\sigma)+\int_{t_{0}}^{t} k(\sigma, s) \omega F^{-1}(v(s)) d s\right] .
$$

Oonsequently, one gets

$$
\varrho^{\prime}(t) \leqslant N(\sigma) k(\sigma, t) \omega I^{-1}(\varrho(t))
$$

It therefore follows that

$$
\Omega(\varrho(\sigma)) \leqslant \Omega\left(\varrho\left(t_{0}\right)\right)+N(\sigma) \int_{t_{0}}^{\sigma} k(\sigma, s) d s .
$$

Hence, the desired result (4.2) follows, noting that

$$
\varrho\left(t_{0}\right)=M(\sigma)+N(\sigma) O(\sigma)
$$

This completes the proof.

A special case of Lemma 1 is also useful in applications which we state as

Coroulary 2. - Assume that $\omega=F$. Then we have the following two different estimates:

$$
\begin{aligned}
& \text { (i) } m(t)+n(t)[o(t)+R(t)] \leqslant[M(t)+N(t) C(t)] \exp \left(N(t) \int_{t_{0}}^{t} k(t, s)\right) d s, \quad t \in I, \\
& \text { (ii) } \quad m(t)+n(t)[e(t)+R(t)] \leqslant \\
& \leqslant m(t)+n(t)\left\{c(t)+\int_{t_{\mathrm{o}}}^{t} k(t, s)[m(s)+n(s) c(s)] \exp \left(\int_{s}^{t} k(s, \theta) n(\theta) d \theta\right) d s\right\}, \quad t \in I .
\end{aligned}
$$

Proof. - The bound givem in (i) follows directly from (4.2) observing that in this case $\Omega(u)=\log u$.

To get the bound in (ii), we have to simply solve the integral equation (4.11) which now takes the form

$$
r(t)=\int_{t_{0}}^{t} k(t, s)[m(s)+n(s)\{c(s)+r(s)\}] d s .
$$


This is accomplished by first letting, for $t_{0} \leqslant t \leqslant \sigma \leqslant T$,

$$
\varrho(t)=\int_{t_{0}}^{t} k(\sigma, s)[m(s)+n(s)\{c(s)+r(s)\}] d s .
$$

Then, noting that $\varrho\left(t_{0}\right)=0$ and solving the linear differential inequality

$$
\varrho^{\prime}(t) \leqslant k(\sigma, t) n(t) \varrho(t)+k(\sigma, t)[m(t)+n(t) c(t)]
$$

which results from (4.5) and the fact that $r(t) \leqslant \varrho(t)$, we readily get (4.4).

Suppose now that $n(t) \equiv 1, c(t) \equiv 0$ and $\omega F^{-1}=g$ is subadditive. Then we have

Lemma 2. - Let $R(t)$ be the maximal solution of

$$
r(t)=\int_{t_{0}}^{t} k(t, s) g(m(s)+r(s)) d s .
$$

Then

$$
\begin{gathered}
R(t) \leqslant \Omega^{-1}\left[\Omega\left(\int_{t_{0}}^{t} k(t, s) g(m(s)) d s\right)+\int_{t_{0}}^{t} k(t, s) d s\right] \\
I_{0}=\left[t \in I \mid \Omega\left(\int_{t_{0}}^{t} k(t, s) g(m(s)) d s\right)+\int_{t_{0}}^{t} k(t, s) d s \in \operatorname{Dom} \Omega^{-1}\right] \quad t \in I_{0},
\end{gathered}
$$

where

$$
\Omega(u)=\int_{\varepsilon}^{u} \frac{d s}{g(s)}, \quad \varepsilon, u>0
$$

Proof. - Let $t_{0} \leqslant t \leqslant \sigma \leqslant T$. Then using the subadditivity of $g$ we get the integral inequality

$$
R(t) \leqslant \int_{t_{0}}^{\sigma} k(\sigma, s) g(m(s)) d s+\int_{t_{0}}^{t} k(\sigma, s) g(R(s)) d s,
$$

which is reducible to the differential inequality

$$
R^{\prime}(t) \leqslant h(\sigma, t) g(R(t))
$$

Therefore integrating from $t_{0}$ to $\sigma$ both sides of the inequality

$$
\frac{d}{d t} \Omega(R(t))=\frac{R^{\prime}(t)}{g(R(t))} \leqslant k(\sigma, t)
$$


we get

$$
\Omega(R(\sigma)) \leqslant \Omega\left(R\left(t_{0}\right)\right)+\int_{t_{0}}^{\sigma} f(\sigma, s) d s .
$$

Thus, noting that $R\left(t_{0}\right) \leqslant \int_{t_{0}}^{\sigma} k(\sigma, s) g(m(s)) d s$ and changing $\sigma$ to $t$ we have the desired result. This completes the proof of Lemma 2 .

\section{5. - Applications.}

In this section, we recover from our results several known results of Gronwall type functional inequalities. We shall begin by deducing the main result of BUTuER and Rogers [2] which, for convenience, we state in the following form.

Theorem 5. - Under the assumptions of Theorem 3 , if $x(t) \in S_{e}$ satisfies the inequality

$$
f(x(t)) \leqslant a(t)+b(t) \int_{t_{0}}^{t} k(t, s) \omega(x(s)) d s
$$

then

$$
x(t) \leqslant f^{-1}[\alpha(t)+b(t) R(t)]
$$

where $R(t)$ is the maximal solution of

$$
r(t)=\int_{t_{0}}^{t} k(t, s) \omega f^{-1}(a(s)+b(s) r(s)) d s
$$

Furthermore,

$$
\begin{gathered}
a(t)+b(t) R(t) \leqslant \Omega^{-1}\left[\Omega(A(t))+B(t) \int_{t_{0}}^{t} k(t, s) d s\right], \\
I_{0}=\left\{t \in I \mid \Omega(A(t))+B(t) \int_{t_{0}}^{t} k(t, s) d s \in \operatorname{Dom} \Omega^{-1}\right\}, \quad t \in I_{0}, \\
A(t)=\sup _{t_{0} \leqslant s \leqslant t} a(s), \quad B(t)=\sup _{t_{0} \leqslant s \leqslant t} b(s)
\end{gathered}
$$

and

$$
\Omega(u)=\int_{\varepsilon}^{u} \frac{d s}{\omega f^{-1}(s)}, \quad \varepsilon, u>0
$$

It is obvious that the proof follows immediately from Theorem 3 and Lemma 1. 
Similarly several other results are readily deducible from our results. We shall indicate some of these without going into details. For instance, to obtain the main result of GoliwItzes [6] set in (3.1) $f(u)=u, \varphi(t)=t, l(t, s) \equiv 1, h(u)=G^{-1}(u)$, $c(t) \equiv 0$, and $\omega(t, u)=\lambda(t) G(u)$. Assume that $G^{-1}$ is convex and sub multiplicative. Suppose there exist continuous functions $\alpha(t)>0, \beta(t)>0$ satisfying $\alpha(t)+\beta(t)=1$ for $t \in I$. Identifying $G$ with $F$ in (3.7), we get from Theorem 4

$$
x(t) \leqslant G^{-1}[m(t)+n(t) R(t)]
$$

where $R(t)$ is the maximal solution of

$$
r(t)=\int_{i_{0}}^{t} \lambda(s) G[m(s)+n(s) r(s)] d s
$$

Moreover, by Corollary 2, (i),

$$
m(t)+n(t) R(t) \leqslant M(i) \exp \left(N(t) \int_{t_{0}}^{t} \lambda(s) d s\right)
$$

and by Corollary 2, (ii),

$$
m(t)+n(t) R\left(t ; \leqslant m(t)+n(t)\left[\int_{t_{0}}^{t} \lambda(s) m(s) \exp \left(\int_{s}^{t} \lambda(\theta) n(\theta) d \theta\right) d \theta\right] .\right.
$$

Similarly, let us set $f(u)=u, b(t) \equiv 1, k(t, s)=\lambda(s), c(t) \equiv 0, \varphi(t)=t, \omega(t, u)=\omega(u)$ and assume that $\omega(u)$ is sub-additive. Then, an application of Theorem 3 gives

$$
x(t) \leqslant a(t)+h(R(t))
$$

where $R(t)$ is the maximal solution of

$$
r(t)=\int_{i_{0}}^{t} \lambda(s) \omega(a(s)+h(r(s))) d s
$$

But, by Lemma 2, we get

$$
R(t) \leqslant \Omega^{-1}\left[\Omega\left(\int_{t_{0}}^{t} \lambda(s) \omega(a(s)) d s\right)+\int_{t_{0}}^{t} \lambda(s) d s\right]
$$

which is precisely the bound obtained by Deo and MURDeshwar in [5]. 


\section{REFERENCES}

[1] I. BHHARI, A generalization of lemma of Bellman and its application to uniqueness problems of differential equations, Acta Math. Acad. Sci. Hung., 7 (1956), pp. 81-94.

[2] G. BUTLER - T. Rogers, A generalization of a lemma of Bihari and applications to pointwise estimates for integral equations, Jour. Math. Anal. Appl., 33 (1971), pp. 77-81.

[3] J. GHANDRA - B. A. FLEISHMAN, On a generalization of the Gronwall-Bellman Lemma in partially ordered Banach spaces, Jour. Math. Anal. Appl., 31 (1970), pp. 668-681.

[4] C. Conduneand, On a class of functional-integral equations, Bull. Math. Soc. Sci. Math. R. S. Roumanie, 12 (1968), pp. 43-53.

[5] S. G. Deo - M. G. Murdeshwar, $A$ note on Gronwall's inequality, to appear.

[6] H. E. Gollwrtzer, A note on a funotional inequality, Proc. Amer. Math. Soc., 23 (1969), pp. 642-647.

[7] L. J. GRIm - K. Schurrт, Boundary value problem for differential equations with deviating arguments, Aequa. Math., 4 (1970), pp. 176-190.

[8] M. A. Kransoserskit, Positive Solutions of Operator Equations, Noordhoff, Groningen, 1961.

[9] V. Lakshmikantham - S. LeEla, Differential and Integral Inequalities, vol. I, Academic Press, New York, 1969.

[10] W. WALteR, Differential and Integral Inequalities, Springer-Verlag, Berlin, 1970. 